\section{P1-266 RELATIONSHIP OF TOOTH LOSS TO MILD MEMORY IMPAIRMENT AND COGNITIVE IMPAIRMENT: FINDINGS FROM THE FUJIWARA-KYO STUDY}

doi:10.1136/jech.2011.142976e.58

\begin{abstract}
${ }^{1} \mathrm{~N}$ Okamoto, ${ }^{*}, 3 \mathrm{M}$ Morikawa, ${ }^{4} \mathrm{~K}$ Okamoto, ${ }^{5} \mathrm{~J}$ Iwamoto, ${ }^{1} \mathrm{~K}$ Tomioka, ${ }^{1} \mathrm{~K}$ Saeki, ${ }^{6} \mathrm{M}$ Yanagi, ${ }^{6} \mathrm{~N}$ Amano, ${ }^{1} \mathrm{~N}$ Kurumatani. ${ }^{1}$ Department of Community Health and Epidemiology, Nara Medical University, Kashihara, Nara, Japan; ${ }^{2}$ Sakai City Mental Health Center, Sakai, Osaka, Japan; ${ }^{3}$ Department of Psychiatry, Nara Medical University, Kashihara, Nara, Japan; ${ }^{4}$ Department of Oral and Maxillofacial Surgery, Nara Medical University, Kashihara, Nara, Japan; ${ }^{5}$ Department of Indoor Environmental Medicine, Nara Medical University, Kashihara, Nara, Japan; ${ }^{6}$ Department of Food and Nutrition, Tezukayama University, Nara, Japan
\end{abstract}

Introduction This cross-sectional study investigated the relationship between the number of remaining teeth to mild memory impairment (MMI), which is a preclinical stage of dementia, and to cognitive impairment.

Methods The subjects were aged 65 years or older and were grouped according to their score for the Mini-Mental State Examination (MMSE), the three-word delayed recall test in the MMSE, and the Geriatric Depression Scale into the control group $(n=3696)$, the MMI group ( $n=121)$, and the low MMSE score (23 or lower) group $(n=214)$. We collected data on the number of remaining teeth, the length of the edentulous period, health-related lifestyle, medical history, blood pressure, height, and body weight. Fasting venous blood samples were also obtained.

Results Multiple logistic regression analysis, adjusted for depressive symptoms, age, sex, length of education, and other explanatory variables, revealed that the ORs of $0-10$ remaining teeth to 22-32 remaining teeth were 1.679 (95\% CI 1.073 to 2.627) for MMI and 2.177 (95\% CI 1.510 to 3.140) for a low MMSE score. A significant relationship was also found between the length of the edentulous period and the risk of a low MMSE score (OR 3.102, 95\% CI 1.432 to 6.720) (15 years or more/<15 years).

Conclusions Our findings suggest that tooth loss is associated with low cognitive function. This possible pathophysiology may be presumed by periodontal disease, gene polymorphisms in inflammatory cytokines, and a decrease in the number of periodontal mechanoreceptors.

\section{P1-267 THE POTENTIAL IMPACT OF SPECIFIC FOOD POLICY CHANGES ON CVD MORTALITY IN IRELAND}

doi:10.1136/jech.2011.142976e.59

${ }^{1} \mathrm{C}$ O'Keeffe, ${ }^{*}{ }^{1} \mathrm{G}$ Browne, ${ }^{2} \mathrm{M}$ O'Flaherty, ${ }^{2} \mathrm{~S}$ Capewell, ${ }^{1} \mathrm{~J}$ Walton, ${ }^{1}$ A Flynn, I I Perry. ${ }^{1}$ University College Cork, Cork, Ireland; ${ }^{2}$ University of Liverpool, Liverpool, UK

Objective To estimate potential reductions in Irish CVD mortality achievable through specific changes in average intakes of saturated fat, trans-fat, salt, fruit and vegetables.

Methods A previously validated food policy model was used to estimate potential annual CVD mortality reductions associated with a conservative scenario. Specifically, a reduction in salt intake by $1 \mathrm{~g} /$ $\mathrm{d}$, trans fat by $0.5 \%$ of energy intake, saturated fat by $1 \%$ energy intake and increasing fruit and vegetable intake by one portion per day. More substantial but politically feasible targets were also modelled. Results were stratified by age and sex. A probabilistic sensitivity analysis was undertaken using Monte Carlo simulation. Results Modest changes in food policy could result in approximately 450 fewer cardiovascular deaths per year. This includes approximately 215 fewer coronary heart disease (CHD) deaths in men (minimum 167, maximum 286), approximately 60 fewer CHD deaths in women $(45,76)$, approximately 115 fewer stroke deaths in men $(92,146)$ and 65 fewer stroke deaths in women $(50,79)$. Approximately, $29 \%$ of the 450 fewer deaths would be attributable to decreased trans fat consumption, $23 \%$ to decreased saturated fat $23 \%$ to decreased salt consumption and $26 \%$ to increased fruit and vegetable consumption. The 450 fewer deaths would represent a $10 \%$ reduction in CVD mortality in Ireland. More substantial but feasible food policy changes could reduce CVD mortality by up to 1250 deaths (representing a $25 \%$ mortality reduction).

Conclusions There are significant opportunities for Government and industry to reduce CVD mortality through evidence-based food policies.

\section{P1-268 ORAL CANDIDIASIS INCIDENCE IN BRAZILIAN HIV INFECTED CHILDREN IN PRE-HAART AND POST-HAART ERAS}

doi:10.1136/jech.2011.142976e.60

${ }^{1} \mathrm{~T}$ C R Oliveira, ${ }^{*} \mathrm{M}$ R D 0 Latorre, ${ }^{1} \mathrm{~L}$ F Tanaka, ${ }^{1} \mathrm{~A}$ M Silva, ${ }^{1} \mathrm{E}$ C Mendes, ${ }^{2} \mathrm{H}$ H S Marques. 'School of Public Health, University of Sao Paulo, Sao Paulo, Brazil; ${ }^{2}$ School of Medicine-University of Sao Paulo Children's Institute, Sao Paulo, Brazil

Introduction Oral candidiasis is the most common opportunistic infection seen in HIV-infected children around the world. The impact of highly active antiretroviral therapy (HAART) has resulted in a decline in the incidence of oral candidiasis in this population. This study aimed to estimate the incidence of oral candidiasis in HIV-infected children in pre-HAART and post-HAART eras.

Methods It was a cohort study. All HIV-infected children admitted in a Brazilian paediatric hospital were followed from 1985 to 2009. For analytical purpose, the patients were divided in two groups according to HIV diagnostic data: pre-HAART (from 1985 to 1998) and post-HAART (1999 to 2009). The incidence rate, incidence rate ratio and CIs were calculated respectively. The incidence rates through people-time were obtained by dividing the number of oral candidiasis episodes by the sum of the years that each subject contributed to the study. Only the first event per patient was considered for the incidence rate.

Results The study included 388 patients. The mean age at admission was 3.3 years and $48.2 \%$ of the children were female. The preHAART and post-HAART incidences of oral candidiasis were 6.4 and 2.7 cases per 100 children-years $(p<0.000)$, resulting in an incidence rate ratio of 2.4 (CI 1.4 to 4.5 )

Conclusion Oral candidiasis incidence was significantly reduced in the post-HAART era in this Brazilian cohort. Continued surveillance is important to assess the long term effect of HAART on the occurrence of opportunistic infections in children.

\section{P1-269 SCREENING FOR BREAST CANCER: ACCESS TO MAMMOGRAPHY IN BRAZIL, 2003-2008}

doi:10.1136/jech.2011.142976e.61

${ }^{1}$ E X G de Oliveira, ${ }^{* 1,2} \mathrm{R}$ S Pinheiro, ${ }^{1,3} \mathrm{E}$ C P Melo, ${ }^{1,4} \mathrm{M}$ Sá Carvalho. ${ }^{1}$ GREI-Saúde/

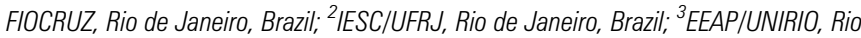
de Janeiro, Brazil; ${ }^{4}$ PROCC/FIOCRUZ, Rio de Janeiro, Brazil

Introduction In Brazil, since 2004, mammography screening for breast cancer is recommended every 2 years for women aged $50-69$. This study examined the effect of external factors-population characteristics and geographic location-on the chances of having a mammogram, in 2003 and 2008.

Methods Patterns of mammography use were examined for women aged 40 years or more as reported in the health supplements of the Brazilian National Household Survey (PNAD) for 2003 and 2008, using multivariate logistic regression. Covariates included were age, race/colour, education, income, self-reported health, insurance, usual source of care, position in the family, having a medical consultation in the last 12 months, urbanisation, and region of residence. 
Results About $52.6 \%$ in 2003 and $67.4 \%$ in 2008 of women aged 40 or older reported receipt of a mammogram. Compared to women 40-49 years old, those aged 50-69 had higher odds of having been screened (1.076 in 2003 and 1.354 in 2008), those aged 70 or older had lower odds (0.513 in 2003 and 0.625 in 2008). The odds increase with family income, education, being married, seeing a physician. Having insurance doubles the odds, as does living in a metropolitan area (3.620 in 2003 and 3.322 in 2008). Compared to the North region, residents in all other regions had larger odds.

Conclusions The age-group targeted by the national policy had marked increase in screening coverage. There are indications of a lessening in inequalities due to income levels, but disparities linked to regional variation have not been reduced.

\section{P1-270 SMALL AND LARGE FOR GESTATIONAL AGE CHILDREN HAVE DIFFERENT EATING BEHAVIOURS AT 6 MONTHS OF AGE}

doi:10.1136/jech.2011.142976e.62

\begin{abstract}
${ }^{1,2} \mathrm{~A}$ Oliveira, ${ }^{*} \mathrm{E}$ Pinto, ${ }^{3} \mathrm{P}$ Moreira, ${ }^{1,2} \mathrm{~A}$ C Santos, ${ }^{1,2} \mathrm{H}$ Barros, ${ }^{1,2} \mathrm{C}$ Lopes. ${ }^{1} \mathrm{Public}$ Health Institute of the University of Porto Medical School, Porto, Portugal; ${ }^{2}$ Department of Hygiene and Epidemiology of the University of Porto Medical School, Porto, Portugal; ${ }^{3}$ Faculty of Nutrition and Food Sciences of the University of Porto, Porto, Portugal
\end{abstract}

Aim To relate eating behaviours at 6 months of age with weight for gestational age at birth.

Methods Study subjects belong to a population-based birth cohort assembled in Porto, Portugal (Generation XXI, $n=8666$ ). A subcohort of 1562 newborns was re-evaluated at 6 months and 1227 singletons presented data on variables of interest. Data were gathered by trained interviewers. Mother answered a questionnaire on socio-demographic, clinical and behavioural characteristics. Small for gestational age (SGA) and large for gestational age (LGA) were defined as $<10$ th and $>90$ th percentile, respectively, of sex-specific Kramer growth charts. OR and $95 \%$ CIs were obtained from unconditional logistic regression, after adjustment for sex, mother's age, education and pre-pregnancy body mass index, maternal smoking during pregnancy, and breastfeeding.

Results Approximately $15 \%$ of children were SGA and 4\% were LGA. Compared to adequate for gestational age children, SGA had more frequently mothers reporting difficulties in feeding at 6 months (OR $=1.52,95 \%$ CI 1.01 to 2.31$)$ and eating small quantities each time (OR=1.78, 95\% CI 1.27 to 2.49). LGA children had also more feeding difficulties ( $O R=2.26,95 \%$ CI 1.10 to 4.63$)$ and a higher probability of refusing solid foods $(\mathrm{OR}=2.21,95 \% \mathrm{CI} 1.02$ to 4.80). No associations were found neither with eating slowly, being angry at the meals' end, choke with food and spitting up milk, nor a later weaning or inclusion of fruits and vegetables as first foods.

Conclusions Both SGA and LGA children presented more feeding difficulties at 6 months of age, and LGA was associated with neophobia to solid foods.

\section{P1-271 PREVALENCE, RISK FACTORS AND PATTERNS OF CHRONIC KIDNEY DISEASE IN A RURAL COMIMUNITY IN SOUTH WEST NIGERIA}

doi:10.1136/jech.2011.142976e.63

${ }^{1} \mathrm{R}$ Oluyombo, ${ }^{*} \mathrm{~A}$ Akinsola, ${ }^{1} 0$ Ayodele, ${ }^{2} \mathrm{~A}$ Onayade, ${ }^{2} \mathrm{~F}$ Arogundade, ${ }^{2} \mathrm{~A}$ Sanusi, ${ }^{1} \mathrm{P}$ Akinwusi, ${ }^{1} 0$ Okunola. 'Ladoke Akintola University of Technology Teaching Hospital, Osogbo, Osun state, Nigeria; ${ }^{2}$ Obafemi Awolowo University Teaching Hospital, Ile-lfe, Osun state, Nigeria

Introduction Chronic kidney disease (CKD) is a global public health problem. Despite the long term difficulties of this condition there is paucity of community derived data in sub-Saharan Africa and especially in Nigeria, the most populated country in Africa. This lack of data is hampering an appropriate response.
Methods Adults (aged $\geq 18$ years) were randomly selected. A structured questionnaire was used to collect data on sociodemographic characteristics and knowledge of kidney disease. Clinical examination was undertaken including: anthropometry, blood pressure, fasting or random blood sugar, dipstick urinalysis, albumin to creatinine ratio and urine microscopy. Glomerular filtration rate (GFR) was estimated using the Modification of Diet in Renal Disease (MDRD) equation.

Results The mean age of participants was $45.8 \pm 19.0$ years with a male: female ratio 0.8:1. 19\% consumed alcohol and 7\% smoked. $20 \%$ used regular analgesia and $75 \%$ used herbal concoctions. The prevalence of hypertension was $30 \%$, diabetes mellitus (DM) (3.7\%), obesity (defined by BMI) $2.7 \%$ and elevated waist circumference (14.6\%). Urine microscopy revealed: haematuria $3.1 \%$, ova of Schistosoma haematobium $1.1 \%$ and macroalbuminuria (8.9\%). An estimated GFR $<60 \mathrm{ml} / \mathrm{min} / 1.73 \mathrm{~m}^{2}$ occured in $12.3 \%$. The prevalence of CKD was $18.8 \%$. Increasing age (OR 0.92, 95\% CI 0.88 to 0.96 ), female gender (OR 4.87, 95\% CI 1.34 to 17.74 ), systolic blood pressure (OR 1.04, 95\% CI 1.01 to 1.07) and DM (OR 15.76, 95\% CI 1.25 to 199.24$)$ were predictive of CKD.

Conclusion $\mathrm{CKD}$ and its risk factors are prevalent in this community. The majority had moderately impaired kidney function. There is need for both primary and secondary preventive programmes.

\section{P1-272 EVALUATION OF EFFECTS OF ATOMIC BOMB SURVIVORS' HEALTH HANDBOOKS ON THEIR HEALTH PROMOTION}

doi:10.1136/jech.2011.142976e.64

K Otani, ${ }^{*}$ M Ohtaki, K Satoh, T Tonda. Hiroshima University, Hiroshima, Japan

Introduction Atomic bombs were dropped on Hiroshima and Nagasaki in 1945, and then A-bomb survivors' health handbooks (shortly 'handbooks') were issued by the Japanese government to help Abomb survivors in 1957. They have been able to receive free medical checkup twice for a year and free medical care for designated disorders. The purpose of the study is to evaluate effects of A-bomb survivors' health handbooks focusing on the relationship between a mortality risk and a length of having handbook.

Methods Objects for analysis were selected from the ABS database of RIRBM Hiroshima University. The number of over-all deaths is 58599 and the number of censored data is 101244 . Cox's proportional hazard model was applied for analysing the data. The observation period is from 1970 to 1997 and the time variable is a time from $1^{\text {st }}$ January 1970 to an occurrence of death. Length of having a handbook was defined as the period from registration year as an A-bomb survivor to 1970. Sex, age at A-bomb exposure, radiation dose and a length of having a handbook are used as covariates.

Results and Conclusion In men, there was significant negative relationship between a length of having a handbook and a relative mortality risk after being adjusted for sex, radiation dose and age at bombing, but in women such a relationship was not found. A man who got a handbook at a young age had a lower mortality risk compared to a man at an old age.

\section{P1-273 THE CHANGING ROLE OF MORTALITY PREDICTORS OVER 20 YEARS OF OBSERVATION}

doi:10.1136/jech.2011.142976e.65

A Pac, ${ }^{*}$ B Tobiasz-Adamczyk, P Brzyski, M Brzyska, M Florek. Jagiellonian University Medical College, Chair of Epidemiology and Preventive Medicine, Krakow, Poland

Longitudinal studies among elderly are concentrated on finding the predictors of mortality. Still, there is an open question if those 